\title{
Commentary: HPV Catch-Up Vaccination Reduces the Prevalence of HPV 16 and 18 Infections and Cervical Disease: A Retrospective Study
}

\author{
Nick F. Hallam ${ }^{1 *}$, Janet A. Parker ${ }^{2}$
}

'Colposcopy Clinic, Women's Outpatients, Cumberland Infirmary, Carlisle, England, United Kingdom

${ }^{2}$ Manchester Cytology Centre, Division of Laboratory Medicine, Manchester University, NHS Foundation Trust, Manchester, England, United Kingdom

\section{Article Info}

\section{Article Notes}

Received: August 25, 2020

Accepted: September 09, 2020

\section{*Correspondence:}

Dr. Nick F. Hallam, Colposcopy Clinic, Women's Outpatients,

Cumberland Infirmary, Carlisle, England, United Kingdom;

Email: Nick.Hallam@uwclub.net

C 2020 Hallam NF. This article is distributed under the terms of the Creative Commons Attribution 4.0 International License.
Human papillomavirus (HPV) vaccination is a success story. Our brief paper (a letter to the editor ${ }^{1}$ ) was a response to the article of Iacobone et al "Distribution of high-risk human papillomavirus genotypes and multiple infections in preneoplastic and neoplastic cervical lesions of unvaccinated women: a cross-sectional study" ${ }^{2}$ in which the authors concluded that the widespread use of prophylactic HPV vaccines could significantly reduce the incidence of preneoplastic and neoplastic cervical lesions. Our study endorsed this statement by describing results from a retrospective cohort study in North Cumbria, England, United Kingdom (UK). The patients involved were two cohorts of women attending for colposcopy after their first cervical screening test. One cohort (tested in 2014-2015) had not been eligible for HPV bivalent (types 16 and 18) vaccination, the other (tested in 2018-2019) was eligible for the HPV bivalent vaccination catch-up campaign. Outcome measures were the HPV primary cervical screening test results and histology results at first colposcopy visit. We found a reduction in the prevalence both of HPV 16 and/or 18 (with/without other high-risk types) from 53\% (103 of 193 patients) to $16 \%$ (23 of 147 patients; $95 \% \mathrm{CI}=27.2 \%-45.6 \%$, $\mathrm{p}<.0001$ ) and of high grade cervical disease (cervical intraepithelial neoplasia 2 or worse) from 54\% (104 of 193 patients) to 23\% (34 of 147 patients; $95 \% \mathrm{CI}=20.8 \%-40.2 \%, \mathrm{p}<.0001$ ) in the bivalent HPV vaccine catch-up 2018-2019 cohort compared with the unvaccinated 2014-2015 cohort. We are currently looking at a third cohort, born from $1^{\text {st }}$ September 1995, who were eligible for routine $\mathrm{HPV}$ vaccination at 12 to 13 years of age.

\section{HPV and cervical disease}

Persistent infection by high-risk HPV types, especially types 16 and 18, is the most important causal factor for the development of cervical precancerous and cancerous lesions. ${ }^{3,4}$

\section{HPV primary screening}

HPV primary screening, whereby HPV testing for high-risk types is the first test done on a cervical screening sample and only HPV positive samples are then triaged by cytology testing, increases the detection of cervical intraepithelial neoplasia grade 3 or worse by approximately $40 \%$ compared to liquid-based cytology. It is currently being introduced across the UK after pilot studies. ${ }^{5}$ Our study was carried out in England where cervical screening starts at 25 years of age whereas in Scotland it used to start at 20 years of age, changing to 25 years of age in June 2016. In practice this means that more followup screening data is available in Scotland compared to England. 


\section{HPV vaccination in the UK}

In the UK, HPV bivalent (types 16 and 18 ) vaccination was introduced in September 2008 for girls aged 12 to 13 years, with a catch-up campaign for girls aged up to 18 years. The policy in the UK is to vaccinate at school where possible rather than through community medical practices and this promotes good uptake. In Scotland, uptake in the routinely immunised cohorts has consistently exceeded $85 \%$, with uptake in the catch-up cohorts at about $65 \%$ overall. ${ }^{6,7}$ The HPV bivalent vaccine was replaced with an HPV quadrivalent one (types 6,11,16 and 18) in September 2012 which was also made available for boys from 2018 and is currently recommended for all girls and boys at 11 to 14 years of age. ${ }^{3}$ The inclusion of low-risk HPV types 6 and 11 adds protection against genital warts. ${ }^{8}$

The bivalent and quadrivalent HPV vaccines confer protection against the vaccine specific HPV high-risk types they contain (types 16 and 18) and also cross protection against additional HPV high-risk types (types 31, 33 and 45). ${ }^{9}$ Together these five types are implicated in $90 \%$ of cancers in Scotland. ${ }^{10}$

Evidence on the impact of HPV immunisation programme showing reduction in HPV type 16/18 infection, genital warts and precancerous lesions among vaccinated cohorts and herd immunity among unvaccinated groups is now emerging globally as well as in the UK..$^{11-18}$

Herd immunity is a significant factor not only for the unvaccinated but also for the unscreened. In one large Scottish study ${ }^{13}$ the attendance for cervical screening at age 20 was a modest $51 \%$ for vaccinated women and a very disappointing $23 \%$ for unvaccinated ones.

\section{Limitations}

In several of the studies cited, including those of Tim Palmer at $\mathrm{al}^{13}$ and ourselves ${ }^{1}$, the results were limited to data from the first year of screening which might lead to an over-estimation of vaccine effectiveness. A further limitation of our study, reflecting the lack of a centralised integrated data base combining vaccination and screening records in England (in contrast to Scotland which has such a system), is that we describe the catch-up cohort as being 'eligible' for HPV vaccination rather than being able to state whether or not they actually received it. In practice herd immunity should have provided some protection for any unvaccinated patients in this cohort.

\section{Conflict of interest}

The authors have declared they have no conflicts of interest. Caldicott Guardian approval (a UK process to ensure that the use of personal data complies with legal requirements for data protection and is in the public interest) was obtained from the Central Manchester University Hospitals NHS Foundation Trust on October,
13, 2016, and from North Cumbria University Hospitals NHS Trust on January 17, 2017, Ref CAL/16/015 for data transfer from the Manchester Cytology Centre to the Colposcopy Clinic at the Cumberland infirmary.

\section{Funding}

None was required.

\section{References}

1. Hallam N, Parker J. HPV catch-up vaccination reduces the prevalence of HPV 16 and 18 infections and cervical disease: a retrospective study. J Low Genit Tract Dis: June 18, 2020 - Volume Publish Ahead of Print - Issue - doi: 10.1097/LGT.0000000000000550

2. Iacobone A, Bottari F, Radice D, et al. Distribution of high-risk human papillomavirus genotypes and multiple infections in preneoplastic and neoplastic cervical lesions of unvaccinated women: a crosssectional study. J Low Genit Tract Dis 2019;23:259-64.

3. Gov.UK. Immunisation against infectious disease. Human papillomavirus (HPV): the green book, chapter 18a. https://www. gov.uk/government/publications/human-papillomavirus-hpv-thegreen-book-chapter-18a

4. Bouvard V, Baan R, Straif K, et al. A review of human carcinogensPart B: biological agents. Lancet Oncol 2009;10: 321-22.

5. Rebolj M, Janet Rimmer J, Karin Denton K et al. Primary cervical screening with high-risk human papillomavirus testing: observational study. BMJ 2019 Feb 6;364:1240. doi:10.1136/bmj.1240.

6. Information Services Division. Scotland. Estimate of HPV vaccine uptake in Scotland, by year of birth. https://isdscotland.scot.nhs. uk/Health-Topics/Child-Health/publications/data-tables 2017. asp?id=2048\#2048.

7. Sinka K, Kavanagh K, Gordon R, et al. Achieving high and equitable coverage of adolescent HPV vaccine in Scotland. J Epidemiol Community Health 2014;68:57-63. doi:10.1136/jech-2013-202620.

8. Barr E and Tamms G Quadrivalent human papillomavirus vaccine. Clin Infect Dis 2007; 45(5): 609-17.

9. Kavanagh K, Pollock K, Cuschieri K, et al. Changes in the prevalence of human papillomavirus following a national bivalent human papillomavirus vaccination programme in Scotland: a 7-year crosssectional study. Lancet Infect Dis 2017;17:1293-302. doi:10.1016/ S1473-3099(17)30468-1.

10. Mesher D, Cuschieri K, Hibbitts S, et al. Type-specific HPV prevalence in invasive cervical cancer in the UK prior to national HPV immunisation programme: baseline for monitoring the effects of immunisation. J Clin Pathol 2015;68:135-40. doi:10.1136/ jclinpath-2014-202681.

11. Cruickshank M, Pan J, Cotton S, et al. Reduction in colposcopy workload and associated clinical activity following human papillomavirus (HPV) catch-up vaccination programme in Scotland: an ecological study. BJOG 2017;124:1386-93.

12. Munro A, Gillespie C, Cotton S, et al. HPV immunisation in a catchup vaccine programme: a two-centre observational study. BJOG 2017; 124:1394-401.

13. Palmer T, Wallace L, Pollock K, et al. Prevalence of cervical disease at age 20 after immunisation with bivalent HPV vaccine at age 12-13 in Scotland: retrospective population study. 2 doi: $10.1136 / \mathrm{bmj} .11161$ | BMJ 2019;365:11161

14. Cameron, R, Kavanagh, K., Pan, J, et al. Human papillomavirus prevalence and herd immunity after introduction of vaccination program, Scotland, 2009-2013. Emerg Infect Dis 2016; 22(1), 56-64. doi:10.3201/eid2201.150736 
15. Innes, C, Sykes, P, Harker, D, et al. Changes in human papillomavirus genotypes associated with cervical intraepithelial neoplasia grade 2 lesions in a cohort of young women (2013-2016). Papillomavirus Res 2018; 6, 77-82. doi:10.1016/j.pvr.2018.10.010

16. Tabrizi, S, Brotherton, J, Kaldor, J, et al. Fall in human papillomavirus prevalence following a national vaccination program. J Infect Dis 2012;206(11), 1645-1651. doi:10.1093/infdis/jis590
17. Tabrizi, S, Brotherton, J, Kaldor, J, et al. Assessment of herd immunity and cross-protection after a human papillomavirus vaccination programme in Australia: a repeat cross-sectional study. Lancet Infect Dis 2014; 14(10), 958-966. doi:10.1016/S1473-3099(14)70841-2

18. Drolet M, Bénard E, Perez N, et al. Population-level impact and herd effects following human papillomavirus vaccination programmes: a systematic review and meta-analysis. Lancet 2019. Epub ahead of print: https:// doi.org/10.1016/S0140-6736(19)30298-3. 